\title{
Editorial
}

\section{Plasma: From Materials to Emerging Technologies}

\author{
Mirosław Dors (D)
}

check for updates

Citation: Dors, M. Plasma: From Materials to Emerging Technologies. Appl. Sci. 2021, 11, 8311. https:// doi.org/10.3390/app11188311

Received: 19 August 2021

Accepted: 6 September 2021

Published: 8 September 2021

Publisher's Note: MDPI stays neutral with regard to jurisdictional claims in published maps and institutional affiliations.

Copyright: (C) 2021 by the author. Licensee MDPI, Basel, Switzerland. This article is an open access article distributed under the terms and conditions of the Creative Commons Attribution (CC BY) license (https:// creativecommons.org/licenses/by/ $4.0 /)$.
Institute of Fluid Flow Machinery, Polish Academy of Sciences, 80231 Gdańsk, Poland; mdors@imp.gda.pl

\section{Introduction}

Interest in plasma as a tool in various technological processes has been growing for several decades. This is because of the special advantage of plasma, which is the immediate generation of chemically active radicals. There are also other advantages of plasma, which depend on its source, e.g., low or high temperature (dielectric barrier discharge vs. plasmatrons), large or small volume (electron beam chambers vs. microplasma), high or low homogeneity (low pressure radio-frequency plasma vs. corona discharge), etc. It is no wonder that plasma is used in so many areas, starting with the synthesis of ozone initiated by Werner von Siemens in 1857, through the activation of material surfaces and flow control by actuators and electrohydrodynamic pumps, to the latest applications related to medicine, environmental protection and stopping climate change.

The objective of this Special Issue is to collect reports on the design and characterization of plasma methods which are or can be used in various types of technologies, especially those that solve contemporary problems regarding materials, energy and the environment.

\section{Review of Issue Contents}

The Special Issue is composed of seven papers covering numerical and experimental research on different aspects related to plasma applications. Here they are introduced in five specific categories that emerge after their reading.

\subsection{Plasma Sources}

Martines et al. [1] designed a helical resonator for radio-frequency plasma generation starting from a theoretical model. The helical resonator is a concept for the production of high voltage at radio frequency, useful for electrical discharges in gases and plasma sustainment. When properly designed, the helical resonator enables the avoidance of the use of a matching network. In this work, researchers consider the treatment of the helical resonator, including a grounded shield, as a transmission line with a shorted end and an open one, with the latter connected to a capacitive load. The input voltage is applied to a tap point located near the shorted end. The authors derived formulas that allowed a prediction of the resonator performance, consequently enabling one to properly design a plasma source according to the parameters required for the plasma. What is important is that the discussion presented in the paper considers the resonator without plasma: the effects of a plasma formed inside the device is going to be the object of a forthcoming publication.

Luo et al. [2] developed a practical method for controlling the switch been the symmetric and asymmetric modes of a dielectric barrier discharge at atmospheric pressure by changing the frequency of the applied voltage. In this work, through a qualitatively validated 1D fluid model, the discharge evolution, manipulating process and underlying mechanism are also presented. It must be noted that the work is a numerical one. The concept is discussed based on simulations. They showed that the effectiveness of the control strategy was determined by the seed electron level at the frequency-altered phase and that there was a critical range of the seed electron density. For example, under the original driving frequency of $14 \mathrm{kHz}$, the seed electron level approximately ranges from $2 \times 10^{13} \mathrm{~m}^{-3}$ to $8 \times 10^{15} \mathrm{~m}^{-3}$. Researchers found, numerically, that the discharges with 
an initial driving frequency of $14 \mathrm{kHz}$ could always be converted to the symmetric mode when the control frequency was beyond $30 \mathrm{kHz}$.

\subsection{Plasma Diagnostics}

Lee et al. [3] investigated the Coulomb-focused bremsstrahlung spectrum resulting from the electron-atom bremsstrahlung process in nonthermal plasma. They derived the universal expression of the electron-atom bremsstrahlung cross section by using the Thomas-Fermi model with the effective charge method, as well as the effective Coulomb focusing factor for the abovementioned process by using the modified Elwart-Sommerfeld factor with the mean effective charge for the binary-encounter. The obtained results should be useful for diagnostics of radiation processes in nonthermal plasma, for hardphoton X-ray spectroscopy, and for diagnostics of fusion plasmas, magnetized plasmas and dusty plasmas.

\subsection{Synthesis of Carbon Structures}

Sobczyk and Jaworek [4] studied the process of growth of various carbon structures in low-current high-voltage electrical microdischarges in argon at atmospheric pressure with an admixture of cyclohexane as the carbon source. The following various types of microdischarges generated at this pressure were tested for both polarities of the supply voltage with regard to their applications to different carbon deposit syntheses: Townsend discharge, pre-breakdown streamers, breakdown streamers and glow discharge. The discharge was generated between a stainless-steel needle and a plate made of a nickel alloy with inter-electrode distances varying between 1 and $15 \mathrm{~mm}$. The results of the experiments carried out at different discharge currents, electrode polarities, inter-electrode distances and cyclohexane concentrations brought the authors to the conclusion that the process of carbon deposition (in the form of carbon nanowalls and carbon microfibers), in particular the morphology of deposits and their growth rate, could be successfully controlled but that the optimal conditions could only be determined experimentally. The paper contains numerous scanning electron microscopy (SEM) pictures of deposits and spectra resulting from the optical emission spectroscopy of plasma formed at different discharge currents.

Li et al. [5] applied microwave plasma at atmospheric pressure to synthesize multiwalled carbon nanotubes on stainless steel by chemical vapor deposition (CVD) using ethanol as a precursor. They compared this method with other low-pressure CVD techniques such as conventional CVD, direct current plasma CVD, radio-frequency plasma CVD and microwave plasma CVD. The obtained results showed that carbon tubes synthesized using atmospheric pressure microwave plasma were characterized by a higher growth rate and lower defects concentration when compared to the conventional CVD, but with similar properties to those produced using other plasma methods. Thus, the advantage of the method applied by Li et al. is that it uses atmospheric pressure instead of reduced pressure. The paper contains pictures of produced carbon structures obtained from SEM and a high-resolution transmission electron microscope as well as Raman and X-ray photoelectron spectroscopy (XPS) spectra.

\subsection{Plasma for Surface Processing}

Krumpolec et al. [6] presented a method for the surface processing of complex polymermetal composite substrates. Atmospheric-pressure plasma etching in pure hydrogen, nitrogen, their mixture as well as in air was used to fabricate flexible transparent composite poly (methyl methacrylate) (PMMA)-based polymer film with Ag-coated Cu metal wire mesh substrates (with conductive connection sites) by the selective removal of the thin $(\sim 10-100 \mathrm{~nm})$ surface PMMA layer. To simulate large-area roll-to-roll processing, the authors used an advanced alumina-based concavely curved electrode generating a thin and high-power density cold plasma layer by the diffuse coplanar surface barrier discharge. After a series of experiments with various gases, it turned out that a short $1 \mathrm{~s}$ exposure to pure hydrogen plasma led to a highly selective etching of the surface PMMA film without 
any destruction of the Ag-coated $\mathrm{Cu}$ metal wires embedded in the PMMA structure. The researchers concluded that the applied direct current surface barrier discharge could be used for the fast, large-area, roll-to-roll and selective plasma etching of complex materials like flexible photovoltaic substrates.

\subsection{Plasma for Exhaust Gas Cleaning}

Cai et al. [7] experimentally studied the process of nitrogen monoxide oxidation (NO) by an atmospheric pressure dielectric barrier discharge (DBD). The subject of the investigation was a gas mixture simulating diesel exhaust from a marine engine. Researchers focused on the effect of electrode parameters such as length, diameter, material and shape. An interesting result was obtained when varying the electrode diameter. Increasing this parameter resulted in a higher oxidation degree of $\mathrm{NO}$ and reduced energy consumption. An increased electrode diameter makes the gas gap of the DBD reactor shorter, which increases the reduced electric field $\mathrm{E} / \mathrm{N}$. As $\mathrm{E} / \mathrm{N}$ increases, the mean electron energy increases as well; thus, increasing the inner electrode diameter makes it easier for the DBD reactor to generate $\mathrm{O}$ radicals and promote $\mathrm{NO}$ oxidation.

\section{Conclusions}

This Special Issue entitled "Plasma: From Materials to Emerging Technologies" covers only very few aspects of plasma applications. For readers this only touches on problems investigated by researchers specialized in plasma physics, chemistry and engineering. There are many scientific teams dealing with other plasma applications not presented here, such as biomedicine, agriculture, tar decomposition in process gas, water remediation soil remediation, food sterilization, hydrogen production, electrostatic precipitating, plasmaassisted combustion, electrohydrodynamics and many more. Fortunately, the Applied Sciences Editorial Board devoted and is going to devote several Special Issues to cover at least part of the research fields mentioned above.

Funding: This research received no external funding.

Acknowledgments: This Special Issues collected the efforts of all the authors, reviewers, and members of the Editorial Office of Applied Sciences. I would like to thank all the professional contributions to this publication.

Conflicts of Interest: The author declares no conflict of interest.

\section{References}

1. Martines, E.; Cavazzana, R.; Cordaro, L.; Zuin, M. The Helical Resonator: A Scheme for Radio Frequency Plasma Generation. Appl. Sci. 2021, 11, 7444. [CrossRef]

2. Luo, L.; Wang, Q.; Dai, D.; Zhang, Y.; Li, L. A Practical Method for Controlling the Asymmetric Mode of Atmospheric Dielectric Barrier Discharges. Appl. Sci. 2020, 10, 1341. [CrossRef]

3. Lee, M.; Ashikawa, N.; Jung, Y. Effect of Coulomb Focusing on the Electron-Atom Bremsstrahlung Cross Section for Tungsten and Iron in Nonthermal Lorentzian Plasmas. Appl. Sci. 2020, 10, 4832. [CrossRef]

4. Sobczyk, A.; Jaworek, A. Carbon Microstructures Synthesis in Low Temperature Plasma Generated by Microdischarges. Appl. Sci. 2021, 11, 5845. [CrossRef]

5. Li, D.; Tong, L.; Gao, B. Synthesis of Multiwalled Carbon Nanotubes on Stainless Steel by Atmospheric Pressure Microwave Plasma Chemical Vapor Deposition. Appl. Sci. 2020, 10, 4468. [CrossRef]

6. Krumpolec, R.; Jurmanová, J.; Zemánek, M.; Kelar, J.; Kováčik, D.; Černák, M. Selective Plasma Etching of Polymer-Metal Mesh Foil in Large-Area Hydrogen Atmospheric Pressure Plasma. Appl. Sci. 2020, 10, 7356. [CrossRef]

7. Cai, Y.; Lu, L.; Li, P. Study on the Effect of Structure Parameters on NO Oxidation in DBD Reactor under Oxygen-Enriched Condition. Appl. Sci. 2020, 10, 6766. [CrossRef] 